favourably with these two large international reports: response rate to ATG+CsA at 6 months was $70 \%$ compared to $35 \%$ for patients receiving CsA alone, $22 \%$ for eltrombopag alone, and $21 \%$ for androgens alone (Figure 1). Overall response rates in patients receiving these different treatment options is outlined in Figure 1, which highlights a superior response rate with the ATG+CsA combination. Figure 1 also shows there was no difference in response for patients in this older patient population receiving horse (hATG) versus rabbit ATG (rATG), which is in keeping with two large recently published real-life studies., ${ }^{7,8}$ Also, survival was comparable in patients receiving horse or rabbit ATG. The higher response rate of patients receiving ATG+CsA did not translate to a significantly improved survival compared to other regimens, as already shown in an EBMT study; ${ }^{9}$ possibly due to second treatment or improved supportive care. Further support to long-term survival also for non-responders comes from the rabbit ATG study; it showed that the 10-year survival of AA patients, classified at 6 months as non-responders to a first course of rATG+CsA, was comparable whether patients were then allografted (64\% survival) or not $\left(60 \%\right.$ survival). ${ }^{8}$ Late responses, improved supportive care, and second treatments could possibly explain the outcome of non-allografted patients.

In conclusion, $\mathrm{ATG}+\mathrm{Cs}$ A remains the treatment of choice for patients with $\mathrm{AA}$, also for those over the age of 60 ; it should be preferred over the administration of CsA with or without androgens or eltrombopag, with or without CsA. Whether the addition of eltrombopag to ATG-CsA first line will further improve the outcome, as recently suggested, ${ }^{10}$ will be determined by an ongoing prospective randomized trial (RACE trial, EBMT). This study also confirms that in real-life analysis, horse or rabbit ATG produce almost identical response rates and survival, also in older AA patients.

\section{References}

1. Contejean A, Resche-Rigon M, Tamburini J et al. Aplastic anemia in the elderly: a nationwide survey on behalf of the French Reference Center for Aplastic Anemia. Haematologica, 2018;104 (2):256-262.

2. Shin SH, Jeon YW, Yoon JH, et al. Comparable outcomes between younger ( $\leqslant 40$ years) and older ( $>40$ years) adult patients with severe aplastic anemia after HLA-matched sibling stem cell transplantation using fludarabine-based conditioning. Bone Marrow Transplant. 2016;51(11):1456-1463

3. Anderlini P, Wu J, Gersten I, et al. Cyclophosphamide conditioning in patients with severe aplastic anaemia given unrelated marrow transplantation: a phase 1-2 dose de-escalation study. Lancet Haematol. 2015;2(9):e367-75.

4. Giammarco S, Peffault de Latour R, Sica S, et al. European Group for Blood and Marrow Transplantation Severe Aplastic Anemia Working Party. Transplant outcome for patients with acquired aplastic anemia over the age of 40: has the outcome improved? Blood. 2018;131(17):1989-1992

5. Al Marki MM, Nathwani N, Yang D, et al. Melphalan based reduced intensity conditioning is associated with favorable disease control and acceptable toxicities in patients older than 70 years with hematologic malignancies, undergoing allogeneic stem cell transplantation. Biol Blood and Marrow Transpl. 2018;24(9):1828-1835

6. Tichelli G, Schrezenmeier H, Socie G, et al. A randomized controlled study in patients with newly diagnosed severe a aplastic anemia receiving antithymocyte globulin (ATG), cyclosporine, with or without GCSF: study of the SAA Working Party of the European Group for Blood and Marrow Transplantation. Blood. 2011;117(17):4434-4441

7. Peffault de la Tour R, Tabrizi R, Marcais A, et al. Nationwide survey on the use of horse antithymocyte globulins (ATGAM) in patients with acquired aplastic anemia: A report on behalf of the French Reference Center for Aplastic Anemia. Am J Hematol. 2018;93(5):1-8

8. Bacigalupo A, Oneto R, Schrezenmeier H, et al. First line treatment of aplastic anemia with thymoglobuline in Europe and Asia: Outcome of 955 patients treated 2001-2012. Am J Hematol. 2018;93(5):643-648

9. Marsh J, Schrezenmeier H, Marin P, et al. Prospective randomized multicenter study comparing cyclosporin alone versus the combination of antithymocyte globulin and cyclosporin for treatment of patients with nonsevere aplastic anemia: a report from the European Blood and Marrow Transplant (EBMT) Severe Aplastic Anaemia Working Party. Blood. 1999;93(7):2191-2195

10. Townsley D, Scheinberg P, Winkle T, et al. Eltrombopag added to standard immunosuppression for aplastic anemia. New Engl J Med. 2017; 376(16):1540-1545.

\title{
Milk and the "Grandmother Effect"- a new contribution to the legacy of Ray Owen
}

\author{
William J. Burlingham \\ University of Wisconsin, Madison, WI, USA \\ E-mail:burlingham@surgery.wisc.edu
}

doi:10.3324/haematol.2018.207340

T he article by Schonewille et al., in the September 2018 edition of Haematologica, ${ }^{1}$ tests the theory originally proposed by Owen et al. [PNAS, 19542] that a "Grandmother Effect" can protect the offspring from hemolytic disease of the fetus and newborn (HDFN) [see illustration, Figure 1]. This disease is caused by maternal antibodies formed against the baby's red blood cells (rbc). One should note here that, thanks to the research of Owen and others in the 1950s, anti-Rhesus $\mathrm{D}(\mathrm{RhD})$ antibody (aka "Rhogam") prophylaxis began to be practiced for all pregnant women who are $\mathrm{RhD}$ - bearing an $\mathrm{RhD}+$ fetus. The absence of such therapy prior to 1954 enabled Owen and colleagues to determine that, when the grandmother had $\mathrm{RhD}$, the mother who was $\mathrm{RhD}$ - and had thereby already been "naturally" exposed to this antigen in utero and as a newborn via breast-feeding, was rendered incapable (for the most part) of producing antibody to an $\mathrm{RhD}$ encountered in her adult life as the mother of a child sired by her $\mathrm{RhD}+$ husband. Subsequent to the routine application of Rhogam (anti-RhD prophylaxis) in the 1960's, the incidence of hemolytic disease of the newborn was greatly decreased, although not completely eliminated. Briefly, Rh- mothers are given a bolus of Rhogam at 28 weeks of pregnancy. If the baby that is born is $\mathrm{Rh}+(\mathrm{a}$ 50:50 chance, if her husband is $\mathrm{RhD}$ heterozyogous), she will receive a second dose, 72 hours after giving birth. This procedure has revolutionized obstetrics and made it possible for healthy $\mathrm{Rh}+$ babies to be born from multiple pregnant $\mathrm{Rh}$ - women.

While a boon to Ob/Gyn medicine, this highly effective clinical practice made it nearly impossible for others to 


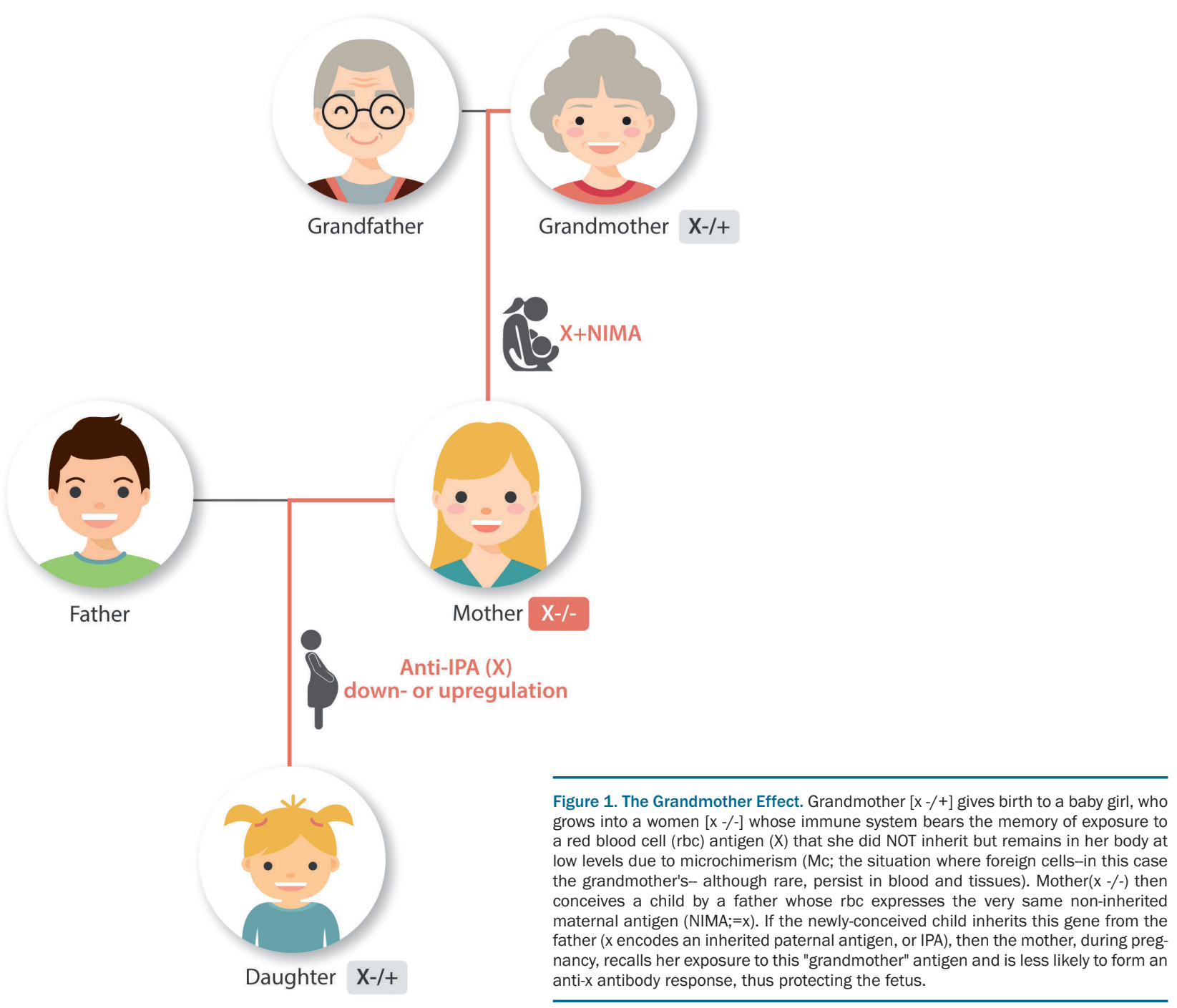

repeat the exact test that Ray Owen had used to develop the Grandmother Effect hypothesis (even though Ray specifically invited others to do $\mathrm{so}^{2}$ ). A torrent of publications followed his, which were not able to confirm his observations of 1954. Meanwhile, other societal changes were occurring, particularly the advent of formula-fed babies, no longer exposed for long periods to breast milk.

Schonewille et al. therefore used a novel approach to the problem, based on the breast-feeding history of the mother provided by the grandmother. The hypothesis was that breast-feeding would condition a baby girl such that antibody response to any non-inherited erythrocyte antigens, including $\mathrm{RhD}$, but also others for which prophylaxis was not done, would be inhibited. When encountered in later life, as she becomes pregnant, they hypothesized that these breast milk exposures from the grandmother would diminish her chances of developing specific antibodies to her husband's (inherited paternal) antigens (IPAs) expressed by her child's rbc.

To test whether there is indeed a cross-generational effect of the grandmother, preventing the daughter from producing antibodies to her baby's rbc and causing hemolytic disease of the newborn, Schonewille et al. chose to study preg-

nancies in cases where mothers had already developed the potential for hemolytic disease. In these women, in the period 1987-2008, their as yet unborn children were being treated by intrauterine transfusion, and their pregnancies managed with the most up-to-date techniques. The investigators studied the antibody responses of the mothers to inherited paternal antigens (IPA) expressed by their babies in relation to various factors, including the breast-feeding history of the maternal grandmother. This approach took the focus off of in utero exposure only and put it onto the combination of in utero exposure and breast-feeding. Schonewille and colleagues made two critically important and novel observations: 1) that there was indeed a Grandmother Effect limiting the mother's ability to produce anti IPA antibodies to a variety of erythrocyte antigens; and 2) that the capacity for a Grandmother Effect was timedependent: i.e., it developed only after at least two months of breast-feeding of the mother by the grandmother. The power of the study was considerable, since the study was done on 125 3-generational Dutch families (grandmothers, mothers, and babies) exposed to 330 non-D rbc antigens and involved the measurement of antibody responses to each of these. Both highly as well as weakly immunogenic 
antigens were studied so as to develop a more balanced model of what the Grandmother Effect might encompass. Out of the $549 \mathrm{rbc}$ antigens not expressed by the mothers (after exclusion for antigens with no exposure from grandmother, or where antigen exposure was unknown), there were 330 known rbc antigen exposures in these women. The differences became meaningful in that, after at least two months of oral NIMA exposure via breast-feeding (i.e., per the maternal grandmother), the odds ratio of the mother (now an adult) forming an antibody to her baby's rbc was very low (0.12). Although continuation of breast-feeding of the grandmother beyond two months did not add additional protections, it was clear that breast-feeding was associated with the protections gained by the NIMA-exposed mother who had been re-exposed to the same antigen as an IPA during her pregnancy.

\section{Discussion}

The observations of Schonewille et al. ${ }^{1}$ add to literature on the NIMA effect in important ways. The original observation of Ray Owen was challenged by a variety of individuals in the Ob/Gyn and blood transfusion fields. However, there was no adequate breast-feeding history of the mothers involved in Ray's study nor of the mothers whose results were claimed to have refuted Owen et al. ${ }^{2}$ However, it was clear from a study by Frans Claas and colleagues in 1988, published in Science, ${ }^{3}$ that in patients awaiting kidney transplant who were highly sensitized to HLA (>90\% reactivity to a random panel), the existence of a NIMA among the different HLA types of cells tested by cytotoxicity assay tended to identify the rare cells to which the highly sensitized patient was unable to make an antibody. Similarly, in 1998 our lab published a paper ${ }^{4}$ showing that the presence of a NIMA HLA haplotype on a sibling kidney allograft donor greatly increased longterm graft survival in the recipient. This was despite the fact that there was an increased rate of early transplant rejection episodes when the NIMA haplotype was present on the haplo-mismatched kidney. ${ }^{4}$ This emphasized the split tolerance nature of the NIMA effect, where certain aspects of cellular immunity, particularly the so-called direct pathway of cellular immunity, were increased by reexposure to the NIMA, whereas the indirect pathway, which controls antibody responses in long-term kidney allograft recipients, was impaired. Some 10 years later, Jeff Mold, Mike McCune and colleagues published a couple of papers, one in $2008^{5}$ and one in $2010,{ }^{6}$ outlining in human studies strong support for the NIMA effect in utero, based on the development of NIMA-specific fetal $\mathrm{T}$ reg populations, and developing a new category for the "normal" CD4 T cell during in utero life-i.e., as one that has " $\mathrm{T}$ reg-like" qualities. Finally, Kinder et al., ${ }^{7}$ working in Sing Sing Way's lab, published a mouse study in 2015 showing exactly how such cross-generational tolerance occurs, and identifying a mechanism whereby NIMA exposure from the grandmother protects the daughter's later pregnancy from fetal loss due to a Listeria infection.

The elegant study by Schonewille and colleagues raises certain questions for the new era of "surrogate" motherhood. First, the surrogate mother is in a completely separate generational lineage, having been exposed by her own mother to certain non-inherited antigens that may or may not match with the antigens of the fetus which she carries. One wonders about the records of successful term pregnancies vs. premature delivery or fetal loss in these "two HLA haplotype mismatched" (fully allogeneic) intrauterine transplants. A second question arising from the study by Schonewille et al. ${ }^{1}$ would be, "Does the surrogate mom breast-feed the baby or not, and if indeed she is breastfed by the surrogate mom, is a baby girl born to the surrogate mom now going to have outcomes of pregnancy that are related to the surrogate mom exposure?" This question can only be answered by further research on these individuals as they become adults. Another question regarding the implications of "milk-kinship" in certain Islamic and Native American societies (en.wikipedia.org/wiki/Milk_kinship), wherein a child is nursed by a woman who is not his/her biologic mother, might now be re-examined in light of the tolerogenic effects of breast-feeding described by Schonewille et al., particularly in those cases where the period of breast-feeding by the "wet nurse" is $\geq 2$ months duration.

\section{Summary}

Overall, the paper by Schonewille et al. ${ }^{1}$ is an important contribution to the literature on the NIMA effect. It is fitting that one of the co-authors of this paper is Jon van Rood, who passed away in 2017. Jon would have been very happy to see this paper published. He was an early champion of the NIMA hypothesis, who shared in the discovery of HLA as a key element in human transplantation, even though the Nobel prize for this was awarded to Jean Dausset. Whether or not the paper promotes a recommendation from Ob/Gyn specialists for two or more months of breast-feeding, it certainly supports such a strategy for female babies, in order to insure long-term protection of babies in the next generation from HDFN.

\section{References}

1. Schonewille H, van Rood JJ, Verduin EP, et al. Exposure to non-inherited maternal antigens by breastfeeding affects antibody responsiveness. Haematologica 2018;104(2):259-264.

2. Owen RD, Wood HR, Foord AG, Sturgeon P, Baldwin LG. Evidence for actively acquired tolerance to Rh antigens. Proc Natl Acad Sci U S A. 1954;40(6):420-424.

3. Claas FH, Gijbels Y, van Der Velden-de Munck J, van Rood JJ. Induction of $B$ cell unresponsiveness to noninherited maternal HLA antigens during fetal life. Science. 1988;241(4874):1815-1817.

4. Burlingham WI, Grailer AP, Heisey DM, et al. The effect of tolerance to noninherited maternal HLA antigens on the survival of renal transplants from sibling donors. N Engl J Med. 1998;339(23):1657-1664.

5. Mold JE, Michaelsson J, Burt TD, et al. Maternal alloantigens promote the development of tolerogenic fetal regulatory $\mathrm{T}$ cells in utero. Science. 2008;322(5907):1562-1565.

6. Mold JE, Venkatasubrahmanyam S, Burt TD, et al. Fetal and adult hematopoietic stem cells give rise to distinct $T$ cell lineages in humans. Science. 2010;330(6011):1695-1699.

7. Kinder JM, Jiang TT, Ertelt JM, et al. Cross-generational reproductive fitness enforced by maternal cells. Cell. 2015;162(3):1-11. 